\title{
Towards a Taxonomy of Ecosystem Data Governance
}

\author{
Dominik Lis \\ Fraunhofer ISST \\ dominik.lis@isst.fraunhofer.de
}

\author{
Boris Otto \\ Fraunhofer ISST \\ boris.otto@isst.fraunhofer.de
}

\begin{abstract}
Despite being in competitive relations, organizations increasingly engage in data-centric collaborations to utilize access and provision to distributed data sources. Over time, these relations have evolved from dyadic relationships to the emergence of complex ecosystems. These ecosystems are characterized by multiple autonomous organizations that engage in data sharing to leverage data-driven innovation. For value propositions based on data to materialize, the configuration of data governance can provide fundamental control mechanisms that influence the design, dynamics and success of the collaboration. In the context of ecosystems, data governance is considered an under-researched topic. This paper investigates both concepts and identifies the main conceptual characteristics of ecosystem data governance. We develop a taxonomy of ecosystem data governance that comprises eight dimensions and twenty four characteristics to improve the conceptual understanding of data governance in data ecosystems.
\end{abstract}

\section{Introduction}

As information technology (IT) investments account for a large share of expenditures in every industry, the penetration of digital technologies within organizations and all areas of society is continuously increasing [1]. This development is accompanied by a paradigm shift towards considering data as a valuable asset. The growing access to diverse data sources creates novel data streams for enhanced utilization of value propositions based on data [2, 3]. Organizations and governments realize the necessity of providing access to data and enabling data sharing for the creation of datadriven services [4].

While data sharing within an organization is considered established practice, sharing data in interorganizational settings is witnessing a global momentum [5,6]. Digital technologies stimulate novel forms of data-centric collaboration between various actors, leading to the emergence of data ecosystems [7, 8], which in turn provide a fertile ground for data-driven innovation, as the collaboration amongst participants fosters access, provision and utilization of distributed data sources [9].

Governance from a data ecosystem perspective in inter-organizational data sharing is beginning to gain more research attention in the information systems (IS) community [10-12]. However, while data ecosystems depend greatly on the provision of data, the role of data governance in this context has not yet been thoroughly investigated. Recent contributions recognize the importance of governance factors in data ecosystems and examine governance as an instrument for facilitating interactions in platform ecosystems [10, 13]. The importance of data governance is emphasized as a fundamental leverage mechanism that influences the design, dynamics and even success of data ecosystems [12].

The exact mechanisms of data governance are not yet fully understood for the participating actors and for the data ecosystem as a whole [8]. While this development has led to a renewed interest in data governance research, the body of knowledge combining these two research streams is still in its infancy [14]. The majority of existing data governance research is focused primarily on an intra-organizational perspective without considering the nature of data ecosystems $[14,15]$.

There is a need to provide structure in the investigation of ecosystem data governance $[10,12,15-$ 17]. We therefore aim at enhancing the conceptual understanding in the application of data governance to complex data ecosystems by identifying the main characteristics of data governance in data ecosystems. This leads to the following research question: What are the main conceptual characteristics of ecosystem data governance? For this purpose, we elaborate on a research design based on the established method for taxonomy development in IS research by Nickerson et al. [18]. The application of this method is recommended to generate consensus among researchers and practitioners on complex domains as in the case of data governance in data ecosystems. To begin this process, we have organized this paper as follows: We first examine the relevant theoretical background of the ecosystem concept and its relation to data governance to 
derive a definition of ecosystem data governance. We proceed with the taxonomy development, which consists of a three-stage plan. First, we identify relevant research articles through a systematic literature review. Second, we draw on the systematic literature review to derive central dimensions and characteristics around ecosystem data governance. Third, we evaluate and discuss the taxonomy to add qualitative grounding for further adjustments and revision of the characteristics. Following this, we present the final taxonomy for ecosystem data governance and apply two cases to test the usefulness and application of our taxonomy. Finally, we discuss findings, implications and conclude the paper with limitations including directions for future research.

\section{Related Work}

\subsection{Ecosystem Concept}

Recent IS literature highlights various concepts around the term "ecosystem", which lead to a high level of ambiguity and fuzziness around central terminology $[19,20]$. The existing concepts around ecosystems have similar principles as they all relate to the concept of biological ecosystems. In general, biological ecosystems can be described as evolving systems that are "dynamic, constantly remaking themselves, reacting to natural disturbances and the competition among and between species" [21]. James Moore [22] first articulated the concept of ecosystems in the field of business environments. He popularized the term business ecosystem to describe new forms of economic activities between businesses to produce "goods and services of value to customers and roles".

The increasing penetration of digital technologies reflects in the analogy of digital ecosystems. Digital ecosystems allow multiple partners to engage in a collaborative network within the correlating technical infrastructure [23]. The focus within the scope of our research are data ecosystems, which can be seen as a subset of digital ecosystems. A universally agreed definition of the term data ecosystem has yet to be established. Here, we follow the definition by [9] who define a data ecosystem as a "... set of interacting actors that directly or indirectly consume, produce, provide data and other related resource". Therefore, the use and sharing of data are key aspects for generating value propositions [9, 24]. Although the emergence and utilization of data ecosystems is still in its infancy, organizations actively engage in the formation of ecosystems to improve their market positioning $[25,26]$. A central constituent of value proposition in this regard is based on data [27]. Providing access to data and using data in the context of inter-organizational data sharing can maximize its economic value [15].

\subsection{Ecosystem Data Governance}

Data governance provides a framework of decisionrights and accountabilities for the management and use of data [28]. It encourages desirable behavior concerning the conduct of data within an organization [29]. The majority of data governance contributions in research and practice are focused primarily on the analysis of single entities. The subject of analysis lies in designing and implementing organizational structures to improve data quality or manage data-related resources across the organization [14].

Recently, scholars have extended the analysis of data governance towards external relationships in ecosystems [12, 15], however, there seems to be no consensus for data governance in intra-organizational settings yet [14, 30]. In the constellation of data ecosystems, the allocation of decision rights and accountabilities to encourage desirable behavior over intangible assets becomes more uncertain [31]. Initial ideas in advancing knowledge have recently begun to examine data governance in the case of platform ecosystems or cloud infrastructures $[10,11,32]$. The vast majority highlights data governance mechanisms and factors associated with a focal key actor and its ecosystem governance. The research attention lies mainly in the analysis of successful platforms owners and their ability to enforce governance mechanisms that influence the dynamics, interactions and success of platforms [11, 33].

While data governance from an intra-organizational perspective implies hierarchical structures and a controllable organizational environment, the structural arrangements regarding data in ecosystems can face tensions between participants involved [15, 34]. The role of ecosystem data governance in this context is to create a collaborative environment that enables data sharing between organizations. It involves establishing coordination mechanisms to ensure the alignment of interests and collective goals between participants. Existing literature provides a limited body of knowledge due to the novel nature in the combination of the two fields. Within the scope of our research we base our understanding of the term ecosystem data governance according to definitions derived from analyses of datacentric collaborations by $[15,31]$. We define ecosystem data governance as "arranged institutions and structures to ensure that individuals behave in line with the collective goals, conflicts between individuals are prevented or resolved, and the effective and fair use of collective resources within the inter-organizational collaboration". 


\section{Research Design}

As our research goal is to identify the main constituents of ecosystem data governance, we employ the established IS research method of taxonomy development. This approach is particularly useful when structuring the knowledge in complex domains to identify common characteristics of the unit of analysis $[18,35]$. Another advantage of using this method for the purpose of our research is that taxonomies assist in bringing structure to detached concepts by merging relevant research streams and examining their relationships [36].

With regard to our research question, we derive common conceptual characteristics of data governance in data ecosystems from literature. The research design comprises three steps. The first step describes the data collection process within the systematic literature review. The goal of this step is to identify relevant research articles around data governance and ecosystems that contribute to providing a basis for the taxonomy development. We screened and selected the papers by analyzing occurring characteristics of data governance and the definition provided on ecosystem data governance.

Subsequently, we develop the taxonomy according to the iterative method proposed by Nickerson et al. [18]. The third step of our research design encompasses an evaluation of our taxonomy based on the analysis of two ecosystem scenarios with data as the core value proposition. The development of the taxonomy is an iterative process, which ends when the defined ending conditions are reached. Figure 1 illustrates the established taxonomy development method in IS research by Nickerson et al. [18].

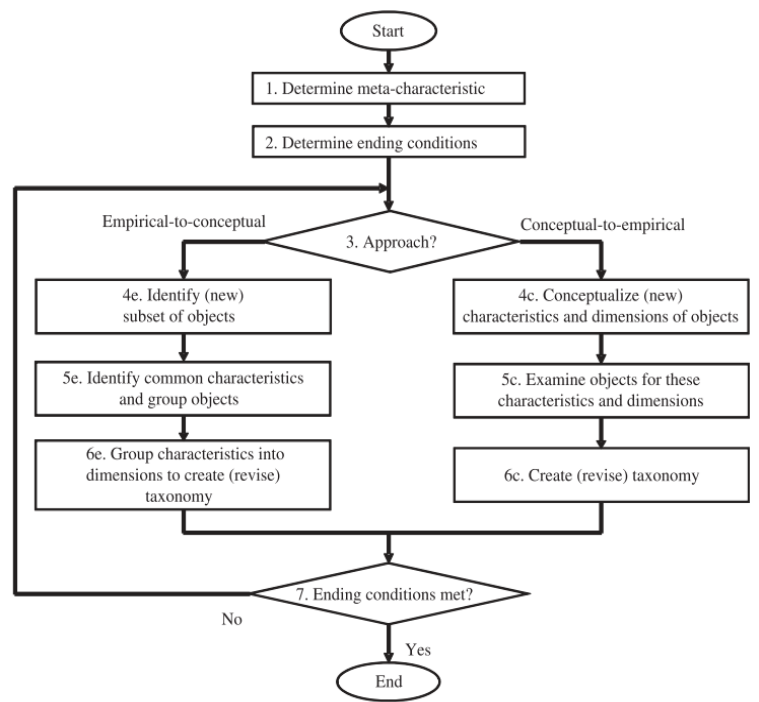

Figure 1. Taxonomy development method

\subsection{Literature Review}

In the first step of our research design, we utilize a structure literature review in compliance with Webster and Watsons' framework [37]. We further considered established guidance by Vom Brocke et al. [38] to support the identification of relevant research in the data governance and ecosystem domain for the taxonomy development. We focused our attention on leading databases that contain literature of high quality from the IS domain. We conducted our literature search within the following databases: AIS Library, ACM Digital Library, IEEE Xplore and Scopus. Due to the scarcity of data ecosystems literature, we decided to use a broader perspective on ecosystems allowing a larger initial literature sample, and used the search string "data governance" AND "ecosystem". We deliberately did not set any restrictions on our search to avoid the exclusion of relevant hits in the rather new combination of these two domains. Due to the scarcity of dedicated data ecosystems literature, we chose a more generic search string.

Our initial search retrieved 161 contributions. Within this specified set, we reviewed the titles and abstracts of the articles and eliminated publications that did not fit our research scope or were not associated with the role of data governance in ecosystems. For instance, we excluded articles that thematize ecosystems from a biological perspective and focused our attention on data ecosystems. As proposed by Webster and Watson [37], we complemented the literature review by applying a backward and forward search for the identification of further relevant publications. This step lead to the consideration of 33 useful contributions which we added to our final literature selection. After eliminating all duplicates, we finally considered 57 articles for the taxonomy development. Figure 2 shows the literature review process.

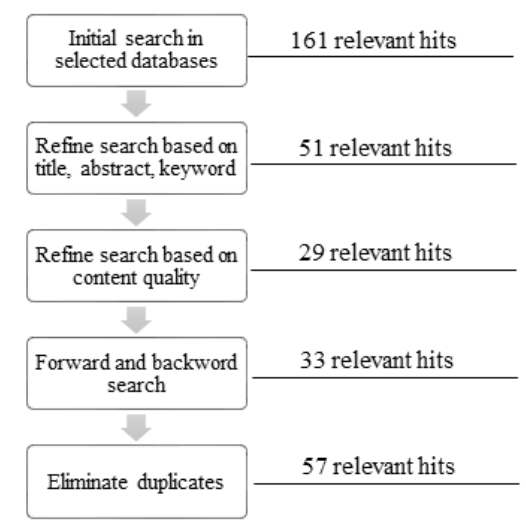

Figure 2. Systematic literature review process 


\subsection{Taxonomy Development}

As depicted in figure 1, the first step of the taxonomy development process by Nickerson et al. [18] is determining a meta-characteristic. The metacharacteristic provides the foundational purpose of the taxonomy, which in our case is the identification of main conceptual dimensions and characteristics of data governance in ecosystems based on the literature review we conducted. Our taxonomy assists researchers and practitioners in structuring relevant research streams and identifying key characteristics.

Due to the iterative approach of the taxonomy development process, it is necessary to define ending conditions. In our case, we aligned our ending conditions with the eight objective and five subjective conditions proposed by Nickerson et al. [18]. After each iteration, we tested the application of the ending conditions to determine when the method was executed sufficiently executed. To reach consensus for the subjective ending conditions concise, robust, comprehensive, extendible and explanatory, we used the questions provided by Nickerson et al. [18] as guidance.

With regard to the objective ending conditions, we engaged in discussions to elaborate on the dimensions and characteristics identified for the taxonomy. The main dimensions and characteristics of our taxonomy are derived from our conducted literature review, and analysis and discussionof selected papers as described in section 3. We chose the empirical-to-conceptual approach as illustrated by phases $4 \mathrm{e}, 5 \mathrm{e}$ and $6 \mathrm{e}$ of the method (see figure 1). We performed four iterations until we met the ending conditions and derived main conceptual dimensions and characteristics for the taxonomy on ecosystem data governance. Our starting point for the first iteration was the analysis of the relevant hits identified from the literature review. After the first iteration, we noted that the overarching setting of ecosystem reflects the classification of governance aspects. We initially added the dimensions Purpose, Configuration and Mechanism with correlating characteristics.

For the second iteration, we further applied the empirical-to-conceptual approach and chose to cluster our analysis in this iteration towards an ecosystemcentric view. Due to the identification a diversity of ecosystem types, we refined our taxonomy by adding additional characteristics and dimensions, namely Scope and Phase. The focus of the third iteration lied in the assessment of governance-centric contributions and as a result, we derived Structure, Data Ownership and Decision Right as relevant dimensions in accordance to our pre-defined meta-characteristc. In

the fourth phase, we chose to validate the identified dimensions and characterstics by applying the conceptual-to-empirical approach. Building on that, we utilized ecosystem governance scenarios based on a convenience sample to improve the application and practicability of the taxonomy. That iteration terminated the method as both objective and subjective ending conditions were met and consensus on the final taxonomy established. The result of the final taxonomy is examined in the next section.

\section{Taxonomy of Ecosystem Data Governance}

The following section describes the constituents of the final taxonomy on ecosystem data governance. As shown in table 1 , the taxonomy consists of eight dimensions with each dimension containing a subset of key characteristics (24 in total). To improve the transparency and understanding of our taxonomy, we grouped the dimensions identified into the three layers on the basis of logical similarity: Interaction, Governance and Data.

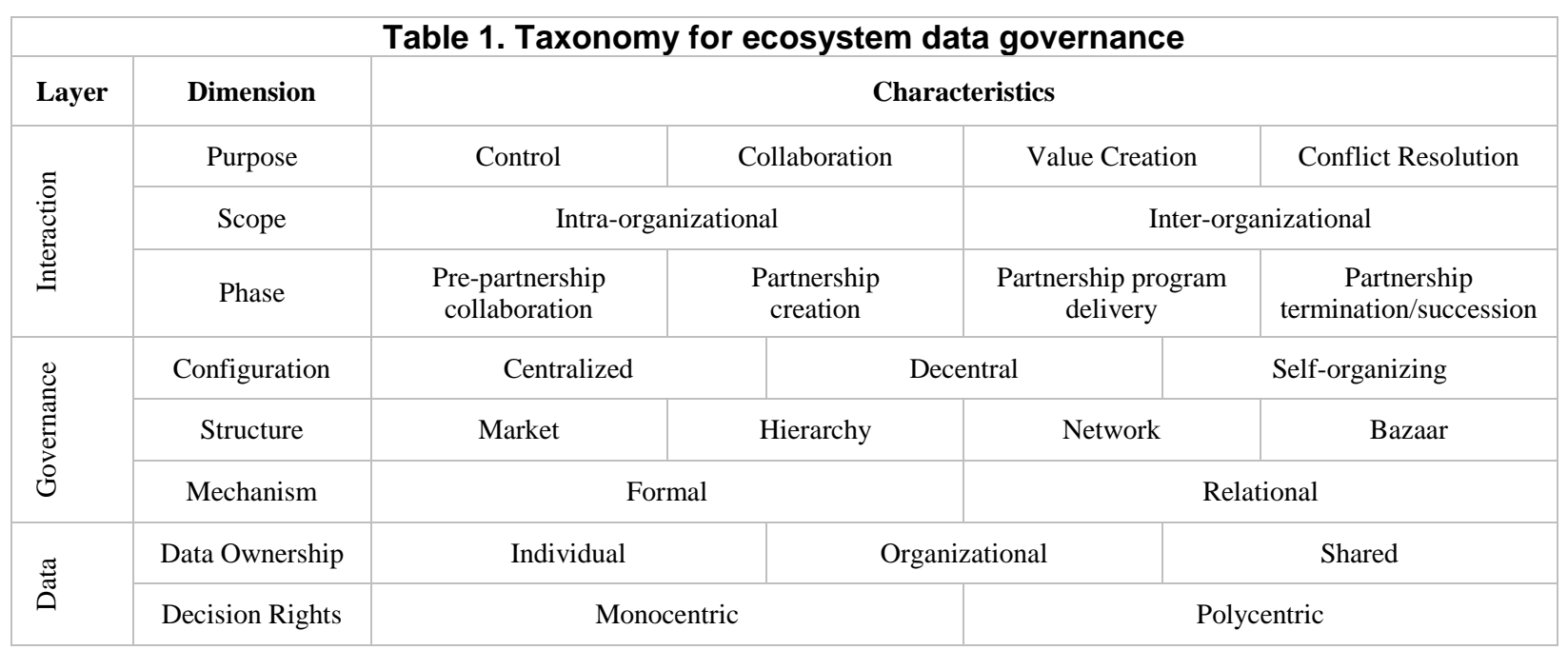




\subsection{Layer 1 - Interaction}

The first layer describes the basic environment of interactions within digital ecosystems. The ecosystem setting encompasses three dimensions: Purpose, Scope and Phase.

Purpose - The purpose dimension covers a subset of four adoption factors that emphasize the need for ecosystem data governance. This dimension encompasses control, collaboration, value creation, and conflict resolution. These characteristics highlight the need for establishing some form of structure to balance collective goals and different interests. In addition, resolving conflicts in inter-organizational firm engagements provides common ground for value creation $[31,33,39]$.

Scope - The scope dimension considers the extent of engagement and participants. We distinguish between an intra-organizational and interorganizational scope [8, 14].

Although ecosystems refer to inter-organizational engagement between multiple firms, the majority of research focuses on the focal actor and its ecosystem governance $[33,38]$. These central actors are capable of setting governance structures within their intraorganizational and controllable environment to manage their respective ecosystems [19]. Sources relate to intraorganizational in the context of managing big data with the adequate software and hardware stack, i.e., software ecosystem or big data ecosystem [40]. The distinction is further required as it affects how and which governance instruments can be deployed to enforce control within a focal firm (e.g., platform owner) and its ecosystem or within the whole ecosystem [8].

Phase - The phase dimension refers to the lifecycle of the collaboration. Four stages of collaboration can be distinguished: Pre-partnership collaboration, partnership creation, partnership program delivery and partnership termination or succession [41]. These collaboration phases affect the potential choice of governance structure and the effectiveness of governance mechanisms throughout the partnership life cycle $[16,38]$.

Pre-partnership describes an early phase of collaboration that evolves around the instantiation of resources and establishing initial requirements. With regard to the emergence of data ecosystems, this phase is characterized by informal governance mechanisms and the intrinsic motivation for collaboration and achieving collective goals [41, 42].

Partnership creation adds formalization to the conduct of collaboration with authority structures and procedures. Partnership program delivery develops towards lower levels of collaboration as regulative market and contractual mechanisms are established.
The final stage of a partnership can either decline towards termination or gain new momentum for succession by maintaining the collaboration and commitment in new governance arrangements [41].

\subsection{Layer 2 - Governance}

The second layer draws upon different governance structures that provide control mechanisms to allow data collaborations in ecosystems. The three dimensions configuration, structure and mechanism encompass mechanisms to align incentives with control mechanisms that can be utilized in ecosystems.

Configuration - Configuration refers to the positioning of the governing body in the ecosystem. It determines the degree to which extent decision-making authority over data can be executed. Dominant actors in the ecosystem, i.e., platform owners who regulate access and interactions within their respective ecosystem, characterize pure centralization.

A decentralized form can be found in settings where the configuration is driven by a shared vision of the community and mutual consensus. Other constructs have self-organizing configurations where the dynamics of the ecosystem follow social socio-technical principles [43]. These self-organized movements are driven by community engagement and share similarities with dynamics in open data initiatives [44, 45].

Structure - The dimension structure describes overarching governance arrangements for control and incentives. Existing literature [41, 46, 47, 48] distinguishes four governance modes: Market, Hierarchy, Network and Bazaar.

These governance modes can be adapted to interpret inter-organizational data collaborations in ecosystems [15]. The market governance mode is characterized by strict compliance with contractual terms over property rights with a low level of trust. A central coordination mechanism within the market mode is pricing [48]. In the context of data ecosystems, market-based arrangements can be seen in data marketplaces with relationships between buyers and sellers of data [15].

The governance mode of hierarchy represents the opposite extreme of a market structure. It is characterized by enforcing control through administrative authority [48]. Hierarchal arrangements imply formal procedures and decisions between individual actors, thus permitting power structures where often dominant actors orchestrate the coordination of collective goals [41].

This mode is visible in supply chain networks where the exchange of data is administrated by dominant actors [15] or in platform settings with platform owners having control over the partnership hierarchy of complementors [31, 39, 46, 49, 50]. 
The network mode of governance was introduced to form a distinct hybrid arrangement setting between the two extreme forms of market and hierarchy [41]. Networks evolve by identifying complementary or collective goals with interdependent capabilites between different organizations. Collaboration in networks is based mainly on reciprocity, common benefits and trust. Decision-making and coordination of the network mode are conducted jointly to reach consensus. The network governance mode shares similarities with multilateral data sharing in data ecosystems or alliance-driven engagements to enable data collaborations $[7,15]$.

The mode of bazaar is an alternative governance structure that was introduced with the emergence of the open source movement. Unlike market or hierarchy structures, bazaar governance is characterized by open licenses that do not require formal contracts or a certain trust basis to enable collaboration [46]. Engagements in the bazaar mode are driven by the willingness to distribute information goods or by intrinsic motivation for better reputation in communities [15]. Our literature search indicates various settings of successful bazaar structures in the context of open data $[4,45,51]$. The public sector has embraced open data initiatives to foster the development of innovative products or services by providing free access to data [52].

Mechanism - The mechanism dimension distinguishes between formal and relational manifestations of governance [53, 54]. It allows the enforcement of control mechanisms that influence the behavior and dynamics of collaboration e.g., through incentives, rewards or penalties $[39,55]$.

Formal regulations provide rules of conduct, i.e., strict development rules that must be obeyed by participating organizations of the ecosystem [56]. Both control rationales are performed in platform ecosystems, whereby platform owners can utilize platform boundary resources like application programming interfaces (APIs) as formal mechanisms to encourage desirable behavior of complementors within the ecosystem [57].

Relational or informal mechanisms are anchored in social norms and constructs to foster adequate behavior. Informal instruments can be used complementary to support formal regulations which is why the types can co-exist and support each other [58]. Relational mechanisms can be grounded in communicating shared visions or values to strengthen the commitment of the ecosystem $[41,59]$.

The informal factor of trust is critical for the success of functional relationships because data is considered a strategically relevant resource by organizations $[60,61]$. Technological design choices and formal agreements can thus be established to resolve conflicts and gain trust $[12,62]$.

\subsection{Layer 3 - Data}

The third layer describes data-related domains consisting of the two distinct dimensions data ownership and decision rights.

Data Ownership - The dimension data ownership is associated with privileges to execute control rights over data access or its usage [63]. Data ownership incorporates related tasks and responsibilities to assure its "fitness for use", respectively aiming at high data quality $[29,64]$. Here, the data ownership dimension indicates the context to which it is assigned. It distinguishes whether data ownership is located at an individual, organizational or shared entity [65].

Data ownership from the individual perspective indicates rights relating to personal data. In the organizational context, data ownership is claimed by an enterprise based on data creation or by engaging in economic activities such as purchasing, licensing or funding data to stake a claim to data ownership. Shared ownership describes a setting without any restrictions over the ownership of data. This characteristic is associated with the open data paradigm, where data is made freely accessible and can be used accordingly by everyone [66].

Decision Rights - The decision rights dimension establishes authority over decision-making for datarelated resources such as determining data standard or usage conditions [62]. In ecosystems, the distribution of decision rights regarding data features monocentric and polycentric characteristics. Polycentricity indicates balance and alignment of decision-rights relying on ecosystem participants for establishing decision-making consensus [17, 30, 66]. Monocentric approaches are centered on focal actors that are capable of cultivating the ecosystem and enforcing decisions $[67,68]$.

\section{Application of the Taxonomy}

This section evaluates the usefulness of the taxonomy we developed and demonstrates its intended purpose as proposed by Nickerson et al. [18]. We apply two cases, which we identified in the course of our literature search and were particularly suitable for further analysis and application to our taxonomy, as they describe data ecosystems in different development stages.

Table 2 provides an overview of how each case fits to the distinct characteristics of the ecosystem data governance taxonomy. 
Case 1: International Data Spaces (IDS) The IDS case is an alliance-driven initiative consisting of researchers and practitioners to develop a platform for secure and trusted data exchange [8, 42]. In case 1 (illustrated by blue dots), the focal points regarding the purpose of interaction in the initial phase is the manifestation of the collaboration and prevention of conflicts at early stages. The interaction focuses on the inter-organizational setting for ecosystem data governance involving multiple and diverse participants.

The research papers at hand $[8,42]$ give insights into the early stages of the development, which is why we placed the blue dot in the pre-partnership collaboration phase. We observe that the development of the IDS has matured in the meantime and now tends to lie in the phase of partnership creation. As mentioned, the phases are overlapping, which is why we placed the dot based on the analysis by [8].

The configuration of the governance structure has central and decentral characteristics. The central entity of the IDS is represented by a not-for-profit association which institutionalizes and oversees decentral development activities. The IDS initiative is driven by core beliefs relating to trust, security and sovereign data exchange. The ecosystem that is formed around the IDS imitative is characterized by reciprocity and common target states and is therefore applicable to the network mode of governance structures.

The IDS case features both formal and relational mechanisms. One formal mechanism is the adherence to organizational and technological requirements provided by a certification body. Relational mechanisms such as communication or knowledge sharing are promoted by the association or single participants of the IDS. Regarding the taxonomy dimensions of data ownership and decision right, the

IDS promotes a data ownership paradigm that enforces data sovereignty. In this way, organizations maintain control over data and define the conditions of its use. With regard to decision-rights for data-related decisions in complex data ecosystems, the IDS includes participants to establish consensus regarding strategic decisions, e.g., orientation towards data standards.
Case 2: Industrial OEM Ecosystem This case is about an established original equipment manufacturer (OEM) specialized in producing industrial machines and plants for the cement industry. The OEM aims to combine physical product expertise with the provision of digital services (e.g., predictive maintenance for better machine utilization) via a platform infrastructure $[7,27]$.

The adaption of case 2 in the taxonomy is illustrated by the red squares. The main purpose of interaction is to utilize machine data for value creation through novel data-driven services. The role of ecosystem governance in this context provides control for the OEM's machineproduced data.

The scope focuses primarily on the intraorganizational perspective of the OEM and its ecosystem governance through the closed analytics platform. As collaboration already exists and is manifested in contractual agreements (long-term buyerseller-relationships regarding the industrial machines), the partnership can be captured within the partnership program delivery phase.

The governance configuration is centralized, as the OEM orchestrates all interactions within the platform. This setting permits the OEM to cultivate its ecosystem in terms of implementing governance mechanisms that effect all users (customers).

The governance structure is characterized by a hierarchy mode, as the centralized configuration allows the orchestration of the data exchange. Access to and usage of the platform is anchored in formal mechanisms such as contracts. Besides contractual arrangements, technical mechanisms with regard to authentication procedures are enforced.

The OEM sees the status of data ownership according to the principle of data creation. Therefore data ownership remains at the organizational level on the customer's side while the OEM leaves it up to the customer whether data is shared for maintenance purposes. The decision-rights in terms of data governance in the data ecosystem rely heavily on the products developed by the OEM, thus following monocentric characteristics.

Table 2. Application of the taxonomy to the selected cases

\begin{tabular}{|c|c|c|c|c|c|}
\hline Layer & Dimension & \multicolumn{4}{|c|}{ Characteristics } \\
\hline \multirow{3}{*}{ 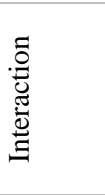 } & Purpose & Control & Collaboration & Value Creation & $\begin{array}{c}\text { Conflict } \\
\text { Resolution }\end{array}$ \\
\hline & Scope & \multicolumn{2}{|c|}{ Intra-organizational } & \multicolumn{2}{|c|}{ Inter-organizational } \\
\hline & Phase & $\begin{array}{l}\text { Pre-partnership } \\
\text { collaboration }\end{array}$ & $\begin{array}{l}\text { Partnership } \\
\text { creation }\end{array}$ & $\begin{array}{l}\text { Partnership } \\
\text { program delivery }\end{array}$ & $\begin{array}{c}\text { Partnership } \\
\text { termination/succession }\end{array}$ \\
\hline \multirow{3}{*}{ 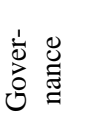 } & Configuration & Centralize & \multicolumn{2}{|c|}{ Decentral } & Self-organizing \\
\hline & Structure & Market & Hierarchy & Network & Bazaar \\
\hline & Mechanism & \multicolumn{2}{|c|}{ Formal } & \multicolumn{2}{|c|}{ Relational } \\
\hline \multirow{2}{*}{ ڤَّ̃ } & Data Ownership & Individual & \multicolumn{2}{|c|}{ Organizational } & Shared \\
\hline & Decision Rights & \multicolumn{2}{|c|}{ Monocentric } & \multicolumn{2}{|c|}{ Polycentric } \\
\hline
\end{tabular}




\section{Conclusion}

The motivation for our research is based on numerous contributions that stress the importance of ecosystem data governance and call for further research in this field $[7,12,19,35]$. The role of ecosystem data governance has become significant to enable datacentric inter-organizational collaborations [8, 12, 42]. The main goal of our study was to bring clarity and structure to disordered research fields by identifying the main conceptual characteristics of ecosystem data governance. We employed the taxonomy development method by [18] and derived eight dimensions and twenty four characteristics for ecosystem data governance. We applied two practical cases of evolving data ecosystems to test the usefulness of the taxonomy. We were able to elaborate both cases along the characteristics of the taxonomy and demonstrated its applicability to structure main the constituents of ecosystem governance.

We acknowledge that our research has limitations. Research on data governance in the context of interorganizational collaborations involving multiple actors is scarce and requires a broader perspective. We suggest further research to investigate how and what data governance arrangements are required to enable dynamic collaborations in ecosystems that go beyond dyadic relationships. It is important to remember that there is still uncertainty regarding the nature and definition of data ecosystems. Furthermore, our taxonomy is based on the data collection derived from the analysis of current literature and verified through the application of practical cases. In some instances, taxonomy design choices may be subject to personal preferences.

Despite its limitations, our research provides contributions to the scientific and managerial knowledge base. From a scientific perspective, our research adds to the understanding of the previously scattered concepts of data governance and data ecosystems. To our knowledge, no study has yet drawn upon the development of a taxonomy to study key characteristics of ecosystem data governance. Researchers can further elaborate on our results to extend and adjust the taxonomy with the knowledge and insights from further practical scenarios. The identification of our dimensions and characteristics regarding ecosystem data governance can be utilized to elaborate governance frameworks specifically to the notion of collaborations in data ecosystems. In this context, adjacent research areas, e.g., the governance of IT [69] with IT systems spanning multiple organizations, may provide helpful insights.

With regard to the managerial perspective, our findings confirm that inter-organizational collaborations in data ecosystems form complex structures with various options to design and enforce governance mechanisms. We note that different social coordination modes can be applied to the notion of collaborations in data ecosystems. Early phases in the emergence of data ecosystems emphasize the advantage of identifying adequate partners to embrace a collective target as in case 1 . To mitigate the risk of growing uncertainty and non-transparent governance structures, formal controls are needed to some extent throughout the collaboration life cycle.

Ecosystem data governance can be strongly influenced by power structures around central actors who provide and cultivate the platform infrastructure as in case 2. This insight is in line with existing research on control authority and influence of dominant actors in ecosystems. The power structure is then shifting towards technical providers capable of executing governance mechanisms within a controllable environment. With the continuous growth of datacentric collaborations, we are expecting more research attention on ecosystem data governance.

\section{References}

[1] Herterich, M. M, Uebernickel, F., and Brenner, W, "Stepwise Evolution of Capabilities for Harnessing Digital Data Streams in Data-Driven Industrial Services", in MIS Quarterly Executive: Vol. 15, Issue 4, Article 6, 2016

[2] McAfee, A., and Brynjolfsson, E., "Big Data: The Management Revolution", Harvard business review 90(10), 2012, pp. 60-68.

[3] Zolnowski, A., Christiansen, T., and Gudat, J., "Business Model Transformation Patterns of Data-Driven Innovations", in Proceedings of the 24th European Conference on Information Systems, 2016.

[4] Enders, T., Wolff, C., and Satzger, G., "Knowing What to Share: Selective Revealing in Open Data", in Proceedings of the 28th European Conference on Information Systems, 2020.

[5] Bertot, J. C., Choi, H., "Big data and e-government: issues, policies, and recommendations", in dg.o '13, 2013.

[6] Nokkala, T., Salmela, H. and Toivonen, J., "Data Governance in Digital Platforms", in Proceedings of the 25th Americas Conference on Information Systems, 2019.

[7] Lis, D., and Otto, B., "Data Governance in Data Ecosystems - Insights from Organizations", in Proceedings of the 26th Americas Conference on Information Systems, 2020.

[8] Otto, B. and Jarke, M., "Designing a multi-sided data platform: findings from the International Data Spaces case", Electron Markets, vol. 29 (4), 2019, pp. 561-580. 
[9] Oliveira, M.I.S., and Lóscio, B.F., "What is a data ecosystem?", in dgo '18, 2018, pp. 1-9.

[10] Lee, S.U., Zhu, L. and Jeffery, R., "Data Governance for Platform Ecosystems: Critical Factors and The State of Practice", in Proceedings of the 21st Pacific Asia Conference on Information Systems, 2017.

[11] Schreieck, M., Wiesche, M., and Krcmar, H., "Design and Governance of Platform Ecosystems - Key Concepts and Issues for Future Research", in Proceedings of the 24th European Conference on Information Systems, 2016.

[12] de Prieelle, F., de Reuver, M. and Rezaei, J., "The Role of Ecosystem Data Governance in Adoption of Data Platforms by Internet-of-Things Data Providers: Case of Dutch Horticulture Industry", IEEE Trans. Eng. Manage, 2020, pp. 1-11,

[13] Hein, A., Schreieck, M., Wiesche, M., and Krcmar, H., "Multiple-Case Analysis on Governance Mechanisms of Multi-Sided Platforms", in Multikonferenz Wirtschaftsinformatik (MKWI), Ilmenau, Germany, 2016.

[14] Abraham, R., Schneider, J., and vom Brocke, J., "Data governance: A conceptual framework, structured review, and research agenda", International Journal of Information Management, vol. 49, 2019. pp. 424-438.

[15] van den Broek, T., and van Veenstra, A.F., "Modes of Governance in Inter-Organizational Data Collaborations", in Proceedings of the 23rd European Conference on Information Systems, 2015.

[16] Markus, M.L. and Bui, Q.N., "Going Concerns: The Governance of Interorganizational Coordination Hubs", Journal of Management Information Systems, vol. 28 (4), 2012, pp. 163-198.

[17] de Reuver, M. and Bouwman, H., "Governance mechanisms for mobile service innovation in value networks", Journal of Business Research, vol. 65 (3), 2012, pp. 347-354.

[18] Nickerson, R.C., Varshney, U., and Muntermann, J., “A method for taxonomy development and its application in information systems", European Journal of Information Systems, vol. 22 (3), 2013, pp. 336-359.

[19] Töpfer, K., Wolfensohn, J.D. and Lash, J., "World Resources 2000-2001, People and Ecosystems: The Fraying Web of Life", Elsevier Science, 2000.

[20] Moore, J.F., "Predators and prey: a new ecology of competition", Harvard Business Review, May-June Issue, 1993.

[21] Immonen, A., Ovaska, E., Kalaoja, J., and Pakkala, D. "A service requirements engineering method for a digital service ecosystem", SOCA, vol. 10 (2), 2016, pp. 151172.

[22] Aarikka-Stenroos, L., and Ritala, P., "Network Vmanagement in the era of ecosystems: Systematic review and management framework", Industrial Marketing Management, vol. 67, 2017, pp. 23-36.

[23] de Reuver, M., Sørensen, C., and Basole, R.C., "The Digital Platform: A Research Agenda", in Journal of Information Technology 33.2, 2018, pp. 124-135.

[24] Attard, J., Orlandi, F., and Auer, S., "Data Value Networks: Enabling a New Data Ecosystem", in IEEE/WIC/ACM International Conference on Web Intelligence, 2016.
[25] Alfaro, E., Bressan, M., Girardin, F., Murillo J., Someh, I., and Wixom, B.H., "BBVA's Data Monetization Journey", MIS Quarterly, vol. 18 (2), 2019, pp. 117-128.

[26] Jacobides, M.G., "In the Ecosystem Economy, What's Your Strategy?", Harvard Business Review 97.5, 2019, pp. 128-137.

[27] Azkan, C., Möller, F., Meisel, L., and Otto, B., "Service Dominant Logic Perspective on Data Ecosystems - A Case Study Based Morphology", in Proceedings of the 28th European Conference on Information Systems, 2020.

[28] Gawer A., and Cusumano, M.A. "Industry Platforms and Ecosystem Innovation", in Journal of Product Innovation Management, vol. 31 (3), 2014, pp. 417-433.

[29] Otto, B., "Organizing Data Governance: Findings from the Telecommunications Industry and Consequences for Large Service Providers", CAIS, vol. 29, 2011.

[30] Weber, K., Otto, B., and Österle, H., "One Size Does Not Fit All - A Contingency Approach to Data Governance", ACM Journal of Data and Information Quality, vol. 1 (1), 2009.

[31] Winkler, T.J. and Wessel, M. "A Primer on Decision Rights in Information Systems: Review and Recommendations", in Proceedings of the 39th International Conference on Information Systems, 2018.

[32] Al-Ruithe, M., Benkhelifa, E., and Hameed K., "Data Governance Taxonomy: Cloud versus Non-Cloud," Sustainability, vol. 10 (2), 2018 pp. 1-26.

[33] Provan, K.G. and Kenis, P., "Modes of Network Governance: Structure, Management, and Effectiveness", Journal of Public Administration Research and Theory, vol. 18 (2), 2007, pp. 229-252.

[34] Selander, L., Henfridsson, O., and Svahn, F., "Capability Search and Redeem across Digital Ecosystems", Journal of Information Technology, vol. 28 (3), 2013, pp. 183197.

[35] Wareham, J.D., Fox, P.B., and Cano Giner, J.L., "Technology Ecosystem Governance", Organization science 25 (4), 2013, pp. 1195-1215.

[36] Glass R.L. and Vessey, I., "Contemporary applicationdomain taxonomies", IEEE Software, vol. 12 (4), 1995, pp. 63-76.

[37] Webster J., and Watson, R.T., "Analyzing the Past to Prepare for the Future: Writing a Literature Review", in MIS quarterly Vol. 26 (2), 2002. pp. xiii-xxiii.

[38] vom Brocke, J., Simons, A., Niehaves, B., and Reimer, K., "Reconstructing the Giant: On the Importance of Rigour in Documenting the Literature Search Process", in Proceedings of the 17th European Conference on Information Systems, 2009.

[39] Dekker, H.C., "Control of inter-organizational relationships: evidence on appropriation concerns and coordination requirements", Accounting, Organizations and Society, vol. 29 (1), 2004, pp. 27-49.

[40] Dinter, B., Gluchowski, P., and Schieder, C., "A Stakeholder Lens on Metadata Management in Business Intelligence and Big Data - Results of an Empirical Investigation", in Proceedings of the 21st Americas Conference on Information Systems, 2015.

[41] Lowndes V., and Skelcher, C., "The Dynamics Of MultiOrganizational Partnerships: An Analysis of Changing Modes of Governance", Public administration, vol.76, Issue 2, 1998. 
[42] Gelhaar, J., and Otto, B., "Challenges in the Emergence of Data Ecosystems", in Proceedings of the 23rd Pacific Asia Conference on Information Systems, 2020.

[43] Basole, R.C., "Visualization of Interfirm Relations in a Converging Mobile Ecosystem", in 7th International Conference on Mobile Business, Jul. 2008, pp. 65-74.

[44] Bonina, C., Eaton, B., and Henningsson, S., "Governing Open Data Platforms to Cultivate Innovation Ecosystems: The Case of the Government of Buenos Aires", in Proceedings of the 39th International Conference on Information Systems, 2018.

[45] Lee, S.U., Zhu, L., and Jeffery, R., "Designing Data Governance in Platform Ecosystems", in Proceedings of the 51st Hawaii International Conference on System Sciences, 2018.

[46] Demil, B., and Lecocq, X., "Neither Market nor Hierarchy nor Network: The Emergence of Bazaar Governance", Organization Studies, vol. 27 (10), 2006, pp. 1447-1466,

[47] Powell, W.W., Staw, B., and Cummings, L.L., "Neither market nor hierarchy", Research in Organizational Behavior, vol. 12, 1990, pp. 104-117.

[48] Williamson, O.E., "The Institutions of Governance", The American Economic Review, vol. 88 (2), 1998, pp. 75 79.

[49] Halckenhaeusser, A., Foerderer, J., and Heinzl, A., "Platform Governance Mechanisms: An Integrated Literature Review and Research Directions", in Proceedings of the 28th European Conference on Information Systems, 2020.

[50] Baijens, J., Helms, R.W., and Velstra, T., "Towards a Framework for Data Analytics Governance Mechanisms", in Proceedings of the 28th European Conference on Information Systems, 2020.

[51] Jetzek, T., Avital, M., and Bjørn-Andersen, N., "Generating Value from Open Government Data", in Proceedings of the 34th International Conference on Information Systems, 2013.

[52] Klievink, B., Neuroni, N., Fraefel, M., an Zuiderwijk, A., "Digital Strategies in Action", in Proceedings of the 18th Annual International Conference on Digital Government Research 2017, pp. 129-138.

[53] Ferguson, R.J., "Contractual Governance, Relational Governance, and the Performance of Interfirm Service Exchanges: The Influence of Boundary-Spanner Closeness", in Journal of the Academy of Marketing Science, vol. 33 (2), 2005, pp. 217-234.

[54] Vandaele, D., Rangarajan, D., Gemmel, P., and Lievens, A., "How to govern business services exchanges: Contractual and relational issues", in International Journal of Management Reviews, vol. 9 (3), 2007, pp. 237-258.

[55] Tiwana, A., Konsynski, B., and Bush, A.A., "Research Commentary-Platform Evolution: Coevolution of Platform Architecture, Governance, and Environmental Dynamics", Information Systems Research, vol. 21 (4), 2019, pp. 675-687.
[56] Manner, J., Nienaber, D., Schermann, M., and Krcmar, H., "Governance for Mobile Service Platforms: A Literature Review and Research Agenda", in International Conference on Mobile Business (IMB), 2012.

[57] Ghazawneh A., and Henfridsson, O., "Balancing platform control and external contribution in third-party development: the boundary resources model", Information Systems Journal, vol. 23 (2), 2013, pp. 173192.

[58] Dyer, J.H., and Singh, H., "The Relational View: Cooperative Strategy and Sources of Interorganizational Competitive Advantage", The Academy of Management Review, vol. 23 (4), 1998, pp. 660-679.

[59] Imperial, M.T., "Using Collaboration as a Governance Strategy", Administration \& Society, vol. 37 (3), 2005, pp. 281-320.

[60] Huber, T.L., Kude, T., and Dibbern, J., "Governance Practices in Platform Ecosystems: Navigating Tensions Between Cocreated Value and Governance Costs", Information Systems Research, vol. 28 (3), 2017, pp. 563-584.

[61] Sarker, S., Sarker, S, Sahaym, A., and Bjørn-Andersen, N., "Exploring Value Cocreation in Relationships Between an ERP Vendor and its Partners: A Revelatory Case Study", MIS Quarterly, vol. 36 (1), 2012, p. 317338.

[62] Van Alstyne, M., Brynjolfsson, E., and Madnick, S., "Why not one big database? Principles for data ownership", Decision Support Systems, vol. 15 (4), 1995, pp. 267-284.

[63] Wang, R.Y., and Strong, D.M., "Beyond Accuracy: What Data Quality Means to Data Consumers", Journal of Management Information Systems, vol. 12 (4), 1996, pp. 5-33.

[64] Fadler, M. and Legner, C. "Who Owns Data in the Enterprise? Rethinking Data Ownership in Times of Big Data and Analytics", in Proceedings of the 28th European Conference on Information Systems, 2020.

[65] Constantinides, P., Henfridsson, O., and Parker, G.G., "Introduction - Platforms and Infrastructures in the Digital Age”, Information Systems Research, vol. 29 (2), 2018, pp. 381-400.

[66] Eaton, B., Elaluf-Calderwood, S., Sorensen, C., and Yoo, Y, "Distributed Tuning of Boundary Resources: The Case of Apple's IOS Service System", MIS Quarterly, vol. 39 (1), 2015

[67] Lee, S.U., Zhu, L., and Jeffery, R., "Data Governance Decisions for Platform Ecosystems", in Proceedings of the 52nd Hawaii International Conference on System Sciences, 2019.

[68] Darking, M., Whitley, E.A., and Dini, P., "Governing diversity in the digital ecosystem", Communications of the ACM, vol. 51 (10), 2008, pp. 137-140.

[69] De Haes, S., and Van Grembergen, W., "IT governance and its mechanisms", Information systems control journal, vol., 2004, pp. 27-33. 